\title{
New records of Microgaster deductor Nixon, 1968 (Hymenoptera: Braconidae: Microgastrinae) for the Holarctic region, with comments on its historical distribution
}

\author{
Jose L Fernandez-Triana ${ }^{\dagger}$ \\ † Canadian National Collection of Insects, Ottawa, and Biodiversity Institute of Ontario, University of Guelph, Ottawa, Canada
}

Corresponding author: Jose L Fernandez-Triana (jftriana@uoguelph.ca)

Academic editor: Lyubomir Penev

Received: 17 Dec 2013 | Accepted: 31 Dec 2013 | Published: 03 Jan 2014

Citation: Fernandez-Triana J (2014) New records of Microgaster deductor Nixon, 1968 (Hymenoptera:

Braconidae: Microgastrinae) for the Holarctic region, with comments on its historical distribution. Biodiversity

Data Journal 2: e1040. doi: 10.3897/BDJ.2.e1040

\section{Abstract}

Four new localities for the species Microgaster deductor Nixon (1968) are recorded from the Nearctic (Canada and the United States) and the Palaearctic (Sweden), expanding significantly the range of the species. $M$. deductor seems to be widely distributed in boreal areas of the Holarctic, and it is characterized by unique morphological (tarsal claw structure) and molecular (COI) traits. Preliminary evidence, to be corroborated when more data is available, suggests that the species might have shifted northwards between 1950 and the present day.

\section{Keywords}

Microgastrinae, Nearctic, Palaearctic, Holarctic distribution, COI gene, morphology, species distribution 


\section{Introduction}

Microgaster deductor Nixon (1968) is a very distinctive microgastrine wasp (Hymenoptera, Braconidae), and can be easily separated from all Holarctic species of Microgaster based on its tarsal claws, which have a lobe (Nixon 1968) (Fig. 2). Until very recently the species was thought to be restricted to northern areas of Europe, in the Western Palaearctic (Huflejt 1997, Koponen and Vikberg 1984). However, a few years ago the species was recorded from Churchill, Manitoba, Canada (Fernandez-Triana et al. 2011, FernándezTriana 2010). That locality was the first record of the species for the Nearctic, and suggested that $M$. deductor could be more widely distributed in sub-Arctic or Arctic areas of the Holarctic (Europe and North America).

Here Microgaster deductor is recorded from four additional localities, three in the Nearctic and one in the Palaearctic, which expand significantly the known range of the species. Morphological and molecular data that serve as diagnostic tools are presented, and the potential of a shift northwards of the species distribution is briefly discussed.

\section{Materials and methods}

All the specimens studied for this paper are deposited in the Canadian National Collection of Insects (CNC), Ottawa, Canada. The only exception is one female deposited in the Biodiversity Institute of Ontario, which was not examined directly. Instead it was studied based on a single photo (habitus, lateral) and associated DNA barcode, both freely available as public data in the Barcode of Life Data Systems (BOLD) (http:// www.boldsystems.org/).

Additionally, 40 specimens of Microgaster spp., representing contemporary collecting from the Swedish Malaise Trap Project (http://www.artdata.slu.se/svenskaartprojektet/ malaisetrap.asp) were examined, although no specimen of $M$. deductor was found in those samples.

The historical data on the distribution of the species was extracted from the original references (Huflejt 1997, Koponen and Vikberg 1984, Nixon 1968) and compared against the new records reported here. A map with the distribution of the species was generated using SimpleMappr (Shorthouse 2010). 


\section{Taxon treatment}

\section{Microgaster deductor Nixon, 1968}

\section{Materials}

Holotype:

a. country: Finland; stateProvince: Lapland; verbatimLocality: Ivalo; individualCount: 1; sex: female; institutionCode: BMNH

\section{Paratypes:}

a. country: Finland; stateProvince: Lapland; verbatimLocality: Ivalo; individualCount: 2; sex: 1 female, 1 male; institutionCode: BMNH

b. country: Sweden; stateProvince: Lapland; verbatimLocality: Torneträsk; individualCount: 1; sex: female; institutionCode: BMNH

\section{Other materials:}

a. country: United States; stateProvince: Alaska; verbatimLocality: Unalakleet; verbatimLatitude: 63.878889; verbatimLongitude: -160.789722; eventDate: 27 Jun 1961, 28 Jun 1961, 4 Jul 1961; individualCount: 3; sex: 1 female, 2 males; recordedBy: Jose Fernández-Triana; institutionCode: CNC

b. country: Sweden; stateProvince: Lapland; verbatimLocality: Abisko; verbatimLatitude: 68.35; verbatimLongitude: 18.816667; eventDate: 29 Jul 1951, 9 Aug 1951, 15 Aug 1951; individualCount: 4; sex: females; recordedBy: Jose Fernández-Triana; institutionCode: $\mathrm{CNC}$

C. country: Sweden; stateProvince: Lapland; verbatimLocality: Abisko; verbatimElevation: 400 m; verbatimLatitude: 68.35; verbatimLongitude: 18.816667; eventDate: 31 Jul 1960; individualCount: 1; sex: female; recordedBy: Jose Fernández-Triana; institutionCode: $\mathrm{CNC}$

d. country: Canada; stateProvince: Northwest Territories; verbatimLocality: Tuktoyaktuk; verbatimLatitude: 66.4445; verbatimLongitude: -133.032; samplingProtocol: Sweeping; eventDate: 14 Jul 2010; individualCount: 1; sex: female; recordedBy: Jose FernándezTriana; institutionCode: CNC

e. country: Canada; stateProvince: Yukon Territory; verbatimLocality: Herschel Island; verbatimLatitude: 69.571; verbatimLongitude: -138.902; eventDate: 29 Jul 2008; individualCount: 1; sex: female; recordedBy: Jose Fernández-Triana; institutionCode: BIO

f. country: Canada; stateProvince: Manitoba; verbatimLocality: $23 \mathrm{~km}$ E of Churchill; verbatimLatitude: 58.734; verbatimLongitude: -93.82; eventDate: 12 Jul 1952, $18 \mathrm{Jul}$ 1952, 23 Jul 1952, 28 Jul 1952, 29 Jul 1952, 3 Aug 1952, 5 Aug 1952; individualCount: 35; recordedBy: Jose Fernández-Triana; institutionCode: CNC

g. country: Canada; stateProvince: Manitoba; verbatimLocality: Warkworth Creek nr. Churchill; verbatimLatitude: 58.375; verbatimLongitude: -93.875; eventDate: 29 Jun 1952, 7 Jul 1952, 3 Aug 1952; individualCount: 6; recordedBy: Jose Fernández-Triana; institutionCode: CNC 


\section{Discussion}

New distribution records for Microgaster deductor in the Nearcitc include three localities: Unalakleet, Alaska (United States), and Tuktoyaktuk, Northwest Territories and Herschel Island, Yukon Territory (Canada). They expand considerably the distribution of the species in the Holarctic, and the Alaskan record suggests the possibility that the species might also be in Siberia, Russia (although that could not be confirmed during this study). The new record in the Palaearctic is from Abisko in Sweden, a locality very close to that of Torneträsk, where one of the paratypes, included by Nixon (Nixon 1968) in his original description of the species, was collected. It should be clarified that Nixon misspelled the name of that locality as Tornekrask.

Considering all the available information for $M$. deductor, the species is relatively widely distributed in Arctic or sub-Artic localities of the Holarctic region, mostly from $59^{\circ}-70^{\circ} \mathrm{N}$ (Fig. 1), and in areas covered by boreal forest, or at the interface of boreal forest with tundra vegetation. The only exception is one female specimen from Poland (Radziejowice near Mszczonów, at $52^{\circ} \mathrm{N}$ ), determined in the 1980's by P. Marczak as belonging to the species (Huflejt 1997). This record might be based on a misidentification of the species, but without examining the specimen it is impossible to re-asses its status.

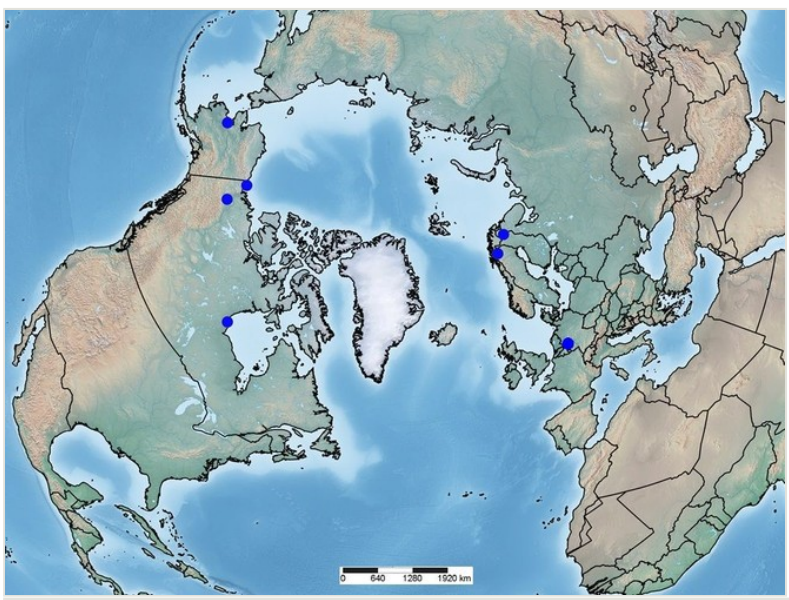

Figure 1.

Distribution of Microgaster deductor.

Morphologically, this species is rather uniform (Figs 2, 3), without any significant external difference between the Palaearctic and Nearctic specimens examined. There is some variation in the pterostigma, which has a pale spot anteriorly in some specimens (e.g., http://www.boldsystems.org/index.php/Taxbrowser Taxonpage?taxon=Microgaster +deductor\&searchMenu=taxonomy\&query=Microgaster+deductor) but in others the pale spot is reduced (e.g., Fig. 3). Since its original description, $M$. deductor has been 
considered as highly aberrant on account of its lobed tarsal claws (Nixon 1968) (Figs 2, 3). No other described species of Microgaster in the Holarctic has this feature. Other diagnostic features are the relatively elongated face, fore wing with vein $r$ strongly inclined towards outer margin of wing, and the shape of the ovipositor sheaths (Figs 2, 3).

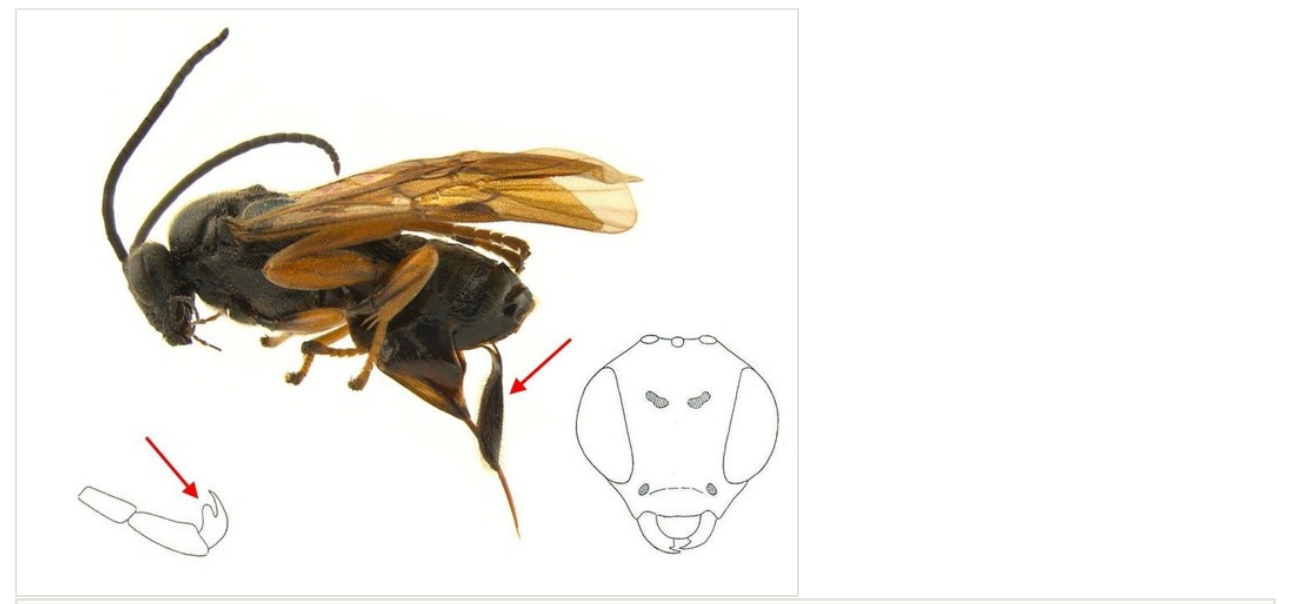

Figure 2.

Microgaster deductor specimen from Churchill, Manitoba. Red arrows show a lobed tarsal claw and the ovipositor sheaths. Frontal view of head modified from Nixon (1968).

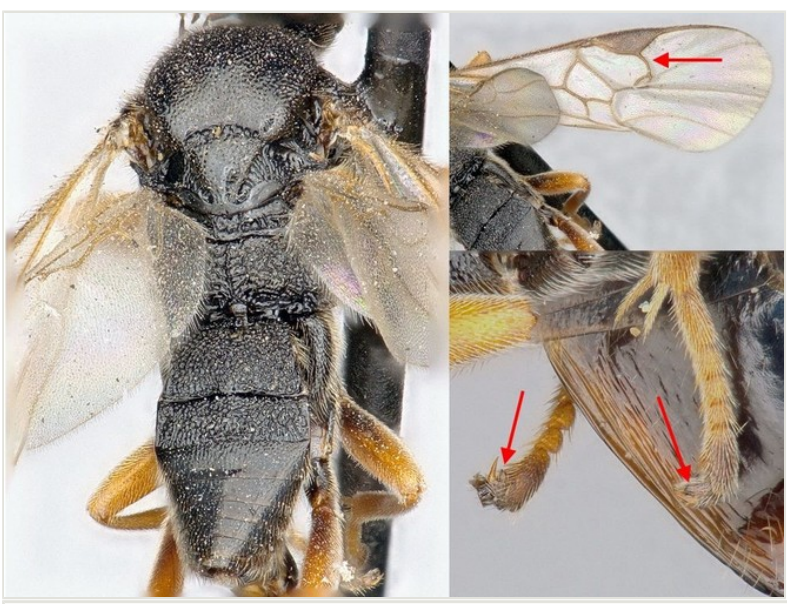

Figure 3.

Microgaster deductor specimen from Abisko, Sweden. Habitus, dorsal view (left), details of fore wing (top right) and tarsal claws (bottom right). Red arrows show tarsal claw lobes, and vein $r$ of fore wing.

The two specimens recently collected (2008 and 2010) rendered full DNA barcodes - a section of 658 base pairs of the mitochondrial COI gene. Among the material collected in Churchill in 1952, one specimen rendered about half a barcode (320 base pairs), and for 
another 33 specimens short sequences of 129-144 base pairs were obtained. All sequences were identical, with the shorter ones perfectly matching the corresponding section of the full barcode obtained from the two recent specimens. The closest sequences in BOLD (which contains a library of over 2.7 million sequences, including more than 800 specimens and 70 species of Microgaster) differed from Microgaster deductor by 46 base pairs $(7 \%)$. Full data of the sequences and specimens can be freely accessed in BOLD from the public projects with codes CNCAS, HARC and WOMIA. The nucleotide sequence of the full barcode of Microgaster deductor, in FASTA format is:

AATATTATATTTTTTATTTGGATTATGATCTGGGATATTAGGATTTTCAATAAGATTAAT TATTCGGTTAGAATTAGGTATTCCTGGTAGATTAATTGGAAATGACCAAATTTATAATAG AATTGTGACATCTCATGCTTTTATTATAATTTTTTTTTATAGTAATACCTGTAATAATTGG GGGATTTGGAAATTGATTAATTCCTTTAATATTAGGTTCTCCAGATATATCATTTCCACG TATAAATAATATAAGATTTTGATTATTAATTCCATCATTAATATTATTAATTTCTAGGAT ATTTATTAATGTGGGTGTTGGAACTGGATGAACAGTTTATCCTCCATTATCATTAATTTT AGGTCATGGAGGTATATCTGTCGATTTAGGAATTTTTTCATTACATTTAGCTGGAGCTTC TTCAATTATAGGTGCAGTAAATTTTATTACAACAATTATAAATATACGAGTTAAAATATA TTTAATAGATAAAATATCTTTATTTTCTTGATCAGTTTTTTATTACTGCAATTTTATTATT AATATCTTTACCTGTTTTAGCAGGTGCTATTACAATATTATTAACTGATCGTAATATTAA TACTAGATTTTTTGATCCTGCTGGTGGAGGGGATCCTATTTTATATCAACATTTATTT

Based on the studied molecular and morphological data, $M$. deductor is a very distinctive and defined species across its whole Holarctic range. At present nothing is known of the Lepidoptera host(s) species that this wasp parasitizes.

The relatively scarce information about historical (1950-1960) and present (2008-2010) distribution of Microgaster deductor, seems to suggest that its range might have shifted northwards recently. For example, the species was the most commonly collected Microgastrinae in Churchill during a period of intense study of insects in the area around the 1950's. However, it has never been found there again, in spite of even more intense collecting efforts carried out in the same locality between 2006-2011 (Fernandez-Triana et al. 2011). Similarly, the species was collected in Alaska, Finland and Sweden between 1950 and 1960, but has not been found later there, in spite of extensive collecting being done in those areas for the past three decades [e.g. Koponen collected 370 specimens of Braconidae in or near the holotype locality in 1983, but did not find M. deductor (Koponen and Vikberg 1984); also, based on the material I have examined so far from the Swedish Malaise Trap Project (including hundreds of Microgastrinae specimens), this project has not recovered the species either]. Conversely, the only two recent records of $M$. deductor correspond to Canadian localities at $69-70^{\circ} \mathrm{N}$, which had similarly been sampled in the 1940-1960 without finding any record of the species. These two localities are $6-10^{\circ}$ north of the Canadian collections from the 1950-1960.

However, both historical and current day distribution patterns are likely to be biased by incomplete sampling efforts. The study of more specimens and more localities will be necessary before reaching any conclusions on this topic. 


\section{Acknowledgements}

The author is very thankful to many fellow colleagues at the Biodiversity Institute of Ontario (Canada) for their support during the past few years, the loan of Arctic material used for this study, and the DNA barcoding of specimens. I am also very thankful to Pelle Magnusson and his colleagues at the Swedish Museum of Natural History in Stockholm and the Station Linné in Öland (Sweden) for sending specimens of Microgastrinae collected as part of the Swedish Malaise Trap Project. The comments of four reviewers improved the final version of the paper.

\section{References}

- Fernandez-Triana J, Smith MA, Boudreault C, Goulet H, Hebert PN, Smith A, Roughley R (2011) A Poorly Known High-Latitude Parasitoid Wasp Community: Unexpected Diversity and Dramatic Changes through Time. PLoS ONE 6 (8): e23719. DOI: 10.1371/journal.pone.0023719

- Fernández-Triana J (2010) Eight new species and an annotated checklist of Microgastrinae (Hymenoptera, Braconidae) from Canada and Alaska. Zookeys 63: 1-53. [In English]. DOI: 10.3897/zookeys.63.565

- Huflejt T (1997) Ichneumonoidea. In: Razowski J (Ed.) Checklist of animals of Poland. Vol. 5: part 32/24 Hymenoptera - postscript. 5. Wydawnictwa Instytutu Systematyki i Ewolucji Zwierząt PAN, Kraków, 260 pp. [In English].

- Koponen M, Vikberg V (1984) Parasitic wasps (Hymenoptera Parasitica) of Inari Lapland, excluding Ichneumonidae. Kevo Notes 7: 101-113. [In English].

- Nixon GE (1968) A revision of the genus Microgaster Latreille (Hymenoptera: Braconidae). Bulletin of the British Museum (Natural History), Entomology series. 22: 33-72. [In English].

- Shorthouse D (2010) SimpleMappr, an online tool to produce publication-quality point maps. URL: http://www.simplemappr.net 